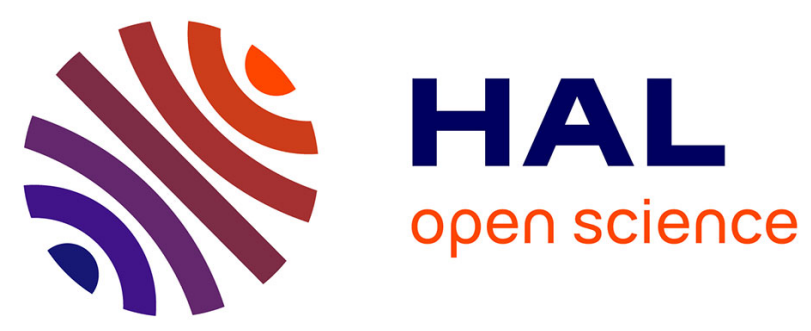

\title{
Broadside FD Antenna Topologies for Nanosat Intersatellite Link
}

Allan Pen, Marc Le Roy, Raafat Lababidi, Denis Le Jeune, André Pérennec, Jean-Luc Issler, Kevin Elis, Adrien Gay, Jean-Hervé Corre

\section{To cite this version:}

Allan Pen, Marc Le Roy, Raafat Lababidi, Denis Le Jeune, André Pérennec, et al.. Broadside FD Antenna Topologies for Nanosat Intersatellite Link. NEWCAS 2020 (18th IEEE International NEWCAS Conference), Jun 2020, Montréal, Canada. pp.1-4. hal-02780479

\section{HAL Id: hal-02780479 \\ https://hal.univ-brest.fr/hal-02780479}

Submitted on 4 Jun 2020

HAL is a multi-disciplinary open access archive for the deposit and dissemination of scientific research documents, whether they are published or not. The documents may come from teaching and research institutions in France or abroad, or from public or private research centers.
L'archive ouverte pluridisciplinaire HAL, est destinée au dépôt et à la diffusion de documents scientifiques de niveau recherche, publiés ou non, émanant des établissements d'enseignement et de recherche français ou étrangers, des laboratoires publics ou privés. 


\title{
Broadside FD Antenna Topologies for Nanosat Intersatellite Link
}

\author{
Allan Pen ${ }^{1,2,3,4}$, Marc Le Roy ${ }^{1}$, Raafat Lababidi ${ }^{2}$, Denis Le Jeune ${ }^{2}$, André Perennec ${ }^{1}$, \\ Jean-Luc Issler ${ }^{3}$, Kevin Elis ${ }^{3}$, Adrien $\mathrm{Gay}^{3}$, Jean-Hervé Corre ${ }^{4}$ \\ ${ }^{1}$ Univ Brest, ${ }^{2}$ ENSTA-Bretagne, Lab-STICC, UMR 6285, CNRS, F-29200 Brest \\ ${ }^{3}$ Centre National d'Etudes Spatiales (CNES), Toulouse ; ${ }^{4}$ Syrlinks, Rennes \\ allan.pen@univ-brest.fr
}

\begin{abstract}
This paper presents and compares four different antenna topologies dedicated to Full-Duplex (FD) applications. The proposed architectures are able to operate simultaneously for transmitting TX and receiving $\mathrm{RX}$ radiating elements along broadside direction, contrarily to most of state of the art FD RF front-ends that also rely on destructive wave's concept to get Self-Interference Cancellation (SIC). The four prototypes make use of three SIC stages and the common first level is obtained using orthogonal linear polarization between $T X$ and $R X$. Step-by-step improvements are introduced from one to another topology in order to increase the SIC level and to reduce both side lobe levels and antenna size. Simulated and experimental results are provided, compared and discussed at the center frequency of $2.4 \mathrm{GHz}$ as a proof of concept. The isolation obtained between TX and $R X$ paths is higher than $80 \mathrm{~dB}$ in simulation and $60 \mathrm{~dB}$ in measurement. These results pave the way to an implementation of a high gain Full-Duplex Ka-band antenna for Nanosat Intersatellite Link
\end{abstract}

Keywords-Full-Duplex, Self-Interference Cancellation, Simultaneous Transmit And Receive (STAR), Inter Satellite Link

\section{INTRODUCTION}

In Band Full-Duplex (FD) consists in transmitting and receiving simultaneously in the same frequency band. The problematic of spectral efficiency has created a need to head on FD communications because in FD it is theoretically possible to operate the same frequency band by 2 users or to allow a double data flow while keeping the same frequency band. Nevertheless, this principle has long been considered impractical due to the high power level of Tx drowning or masking the lower level signal to be received at $\mathrm{Rx}$. Recent studies have opened the way to practical realizations of FD [1]-[3] but for narrowband transmissions and at short distances. The main challenge comes from the very high level of self-interference (SI) which is the result of a high level of power TX leaking into the RX. The greater the channel distance between the two FD antenna systems, the higher the required isolation level. A simple link budget calculation reveals that total transmit/receive isolation levels in the range of 110-130 dB between transmit and receive chains may be required depending on applications. To achieve this level of isolation, successive stages of separation and cancellation among the antenna, RF, analog and digital levels are needed. Recent studies have pointed their efforts on obtaining the best possible isolation between $\mathrm{Tx}$ and $\mathrm{Rx}$ at the antenna stage by generating $\mathrm{Tx}$ waves that interact destructively at the spot where the Rx antenna is located [1]-[3]. This $1^{\text {st }}$ level of
SIC (Self-Interference Cancellation) is of prime interest so as not to degrade and saturate the dynamic ranges of the RF components of the Rx chain and as a priority the ADC one. The desired final SIC is generally reached by a complementary final digital correction stage. Indeed, the algorithms used make it possible to obtain a particularly efficient adaptive SIC in a changing environment to take into account multiple paths (NLOS).

Our objective is to study the feasibility of a FullDuplex transmission between nano-satellites [4] (ISL: InterSatellite-Link). In this context, the desired total SIC level is very important, of the order of $110-130 \mathrm{~dB}$ at least, directly dependent on the distance between satellites. As a counterpoint to this rough specification, NLOS in such a Low Earth Orbit (LEO) ISL link context will be minimized compared to indoor-channel communication. This boost the interest of having a significant level of SIC at the $1^{\text {st }}$ antenna/RF stage. Whereas most previously proposed topologies do not allow RX and TX antennas to operate in the same axial direction (i.e. broadside direction for planar antennas), a recent study manages to overcome this limitation in the case of circular polarization [5]. We propose in this paper to compare different antenna configurations that allow having constructive waves in far field from the separate Tx antennas (as for classical antenna array) while having a destructive behavior in near-field on the Rx antennas. An identical and complementary step is applied at the Rx level. The following section will first illustrate and describe the concept described above. In section III, 4 different antenna configurations with different feeding techniques will be compared in simulation and experimentally and the results will be discussed in order to identify the different ways of improving the SIC. The knowledge gained through these low-cost circuits implementation will serve to design more advanced prototypes dedicated to Ka-Band ISL link.

\section{Principe Of Self-Interference CANCELlation AT the ANTENNA LEVEL}

The major considerations toward a full-duplex antenna front-end design consist in achieving a sufficient SIC level and also to have a main lobe radiation pattern expected along the same direction for Rx and Tx. Moreover, the topology must be compatible with multiple antennas (antenna array) both for TX and RX in order to get a high gain with a narrow directive beam as required for the targeted application. The initial concept of signal nulling for FD applications has been introduced in [1]-[3]. The idea proposed in these topologies relies on a pair of emitting elements which are radiating with identical magnitude but with opposite phase. The receive elements are placed on the median axis perpendicular to the TX antennas where the TX waves destructively add. The residual SI is suppressed by 


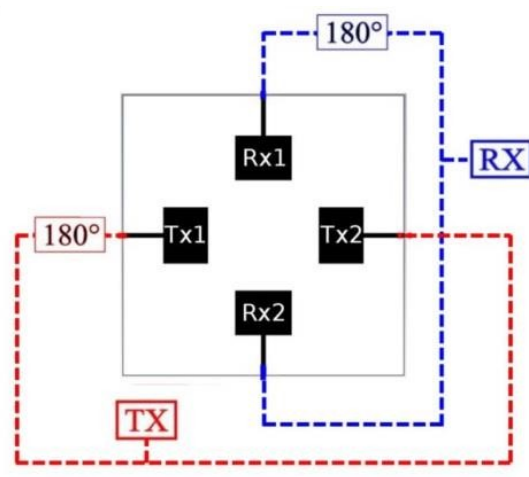

Fig. 1. Broadside FD antenna principle.

using a complementary architecture in the RX path. Contrarily to other techniques, the SIC bandwidth limitation is completely uniquely correlated to the phase shifter or inverter bandwidth.

Although the concept put forward in [1]-[3] is able to reach high level of SIC, both the RX and TX main lobes face a null plane in broadside direction, i.e. the main beam is divided into two symmetrical lobes.

Our goal is to design a topology able to deal with the high level of SI and to cancel it in near field, whereas combining the TX and RX signals in far field in the normal direction to the antenna plane. Recent works and studies [4] have pave the way for such expectations. Fig. 1 illustrates the proposed concept based on 4 elements which are sequentially rotated by $90^{\circ}$, i.e. it means that the physical antenna feedings are also sequentially offset from $90^{\circ}$. So, each pair of antennas oriented face to face are respectively dedicated to the transmitting part and receiving part; and within these antenna pairs, the antennas are oppositely fed. Then, using the same technique as MIDU [3], the face-to-face antennas are fed with identical magnitude but in opposite phase. Therefore, as evidenced in Fig. 2.a, there is a null E-field axis across the RX antennas when the TX antennas are fed as described previously, together with a constructive farfield (contrarily to [2]) as illustrated by the corresponding radiation pattern of Fig. 2.b.

In our topology, three levels of antenna cancellation are introduced:

i) The first stage of SIC is due to the cross polarization between the RX and TX positioning of antennas.

ii) Opposite physical feeding point of TX antennas associated with an emitted signal fed with identical amplitude and opposite phase provides a $2^{\text {nd }}$ level of SIC

iii) Finally, the residual components received on $\mathrm{RX}$ antennas, despite the two first SIC levels, are combined destructively by using a reciprocal architecture within the $\mathrm{RX}$ path. It should pointed out that this receiving path does not suppress the received useful signal but only the nearfield SI components, following the same mechanism as for TX.

The following section will compare different configurations in order to both increase the level of SIC and improve the realized gain in the desired direction by reducing the sidelobes. (a)

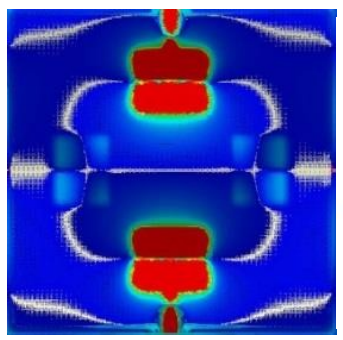

(b)

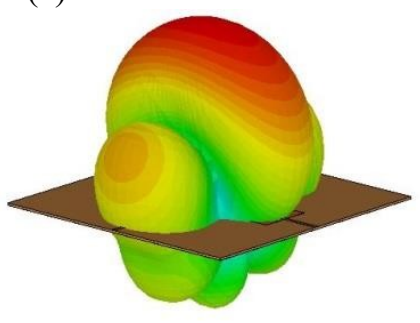

Fig. 2. (a) Electric near-field distribution for the initial architecture, and (b) illustration of the corresponding 3-D radiation pattern.

\section{Simulated AND EXPERIMENTAL BenCHMARK OF BROADSIDE FD ANTENNA TOPOLOGIES}

This section details the 4 tested configurations and propose benchmark of SIC and radiation pattern performance from simulated and measured results. Antennas and baluns (a power divider with an inverter in one of the output branches) were created by using a low-cost and simple technological process, in this case a LPKF PCB prototyping machine and on a $1.6 \mathrm{~mm}$ thick FR-4 substrate. Moreover, the circuits were designed to operate around 2.4 $\mathrm{GHz}$ in order to limit connection issues as well as parasitic, VNA calibration, and second-order effects at this proof-ofconcept step. All the topologies were designed on CST Studio simulator by following the principle described in section II but different feedings and placements of the antennas on the substrate are used. Four possibilities (Fig. 3) are introduced by i) either implementing microstrip or coaxial feedings; and also ii) by either horizontally and vertically aligning the TX and RX antennas or along diagonal axes. The last proposal aims at designing a more compact antenna architecture, theoretically more interesting for sidelobe level reduction. In all these simulated results, classical microstrip out-of-phase power dividers were used as illustrated in Fig. 3. Losses were taken into account in all simulations.

Fig. 3 shows the simulated S-parameters of the 4 configurations where $S_{21}$ mimics the SIC level or isolation level. The antenna matching and the isolation levels are of the same order of magnitude for all topologies, typically better than $85 \mathrm{~dB}$ for isolation in the operating band. The corresponding radiation patterns are plotted in Fig. 4 at $\Phi=90^{\circ}$.

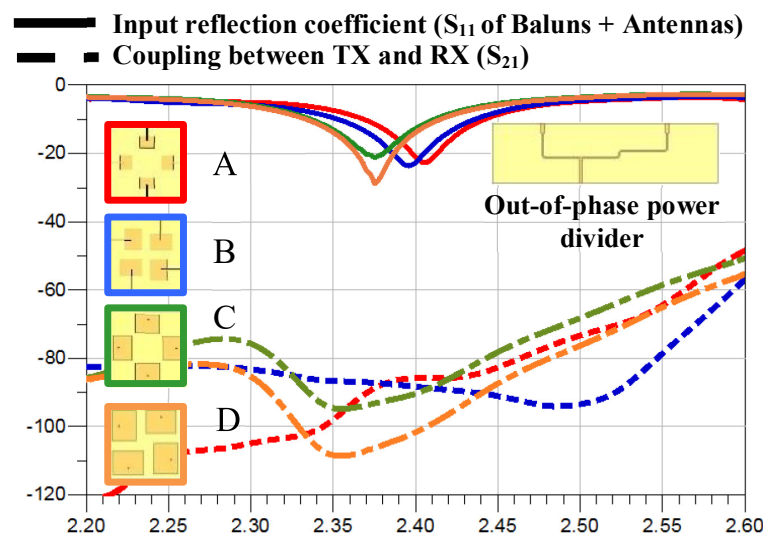

Fig. 3. Simulated input reflection coefficient $\left(\mathrm{S}_{11}\right)$ and coupling between TX and RX $\left(\mathrm{S}_{21}\right)$ for the four architectures of antennas using microstrip outof-phase power dividers. 

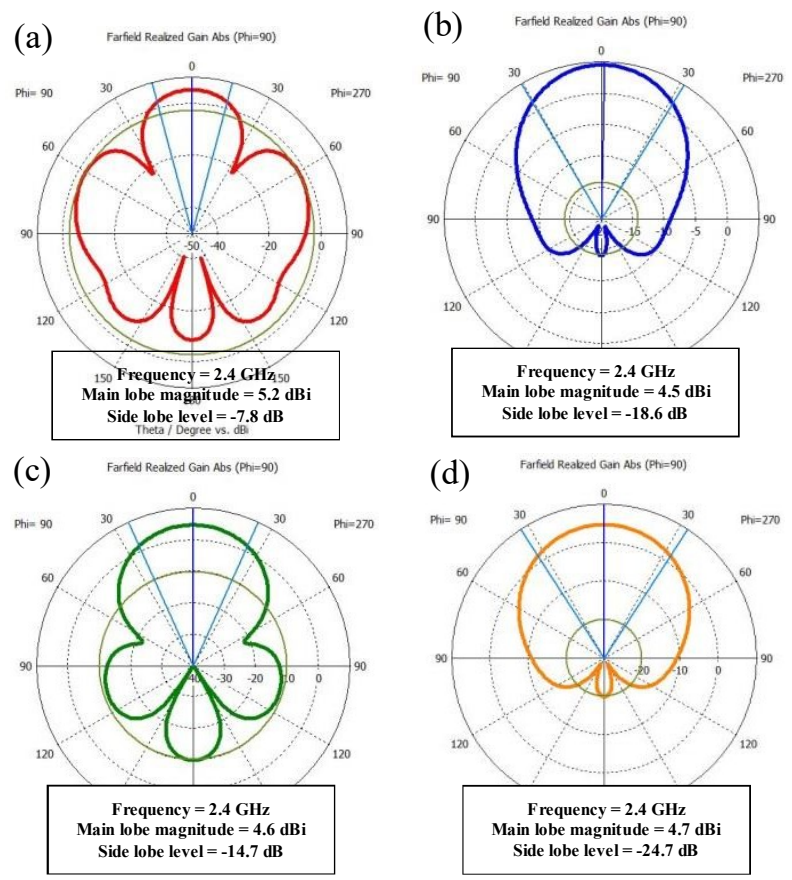

Fig. 4. Simulated Radiation pattern at $2.4 \mathrm{GHz}$ of the four antenna topologies, (a) topology A: using microstrip feeding line and antennas placed face to face; (b) topology B: using microstrip feeding line and antennas in diagonal configuration; (c) topology C: using coaxial feeding and antennas placed face to face; (d) topology D: coaxial feeding with antennas in diagonal configuration. (a)

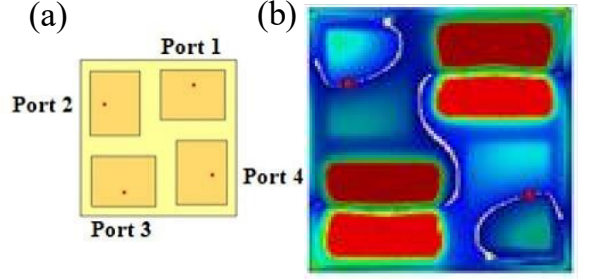

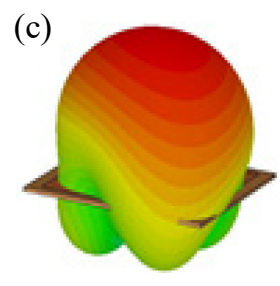

(c)
Fig. 5. (a) Antenna ports, (b) Electric field distribution for architecture D, and (c) illustration of the corresponding 3-D radiation pattern.

Fig. 3 evidenced a slight improvement in the isolation level while using coaxial feeding and also more when the antenna get closer (i.e. Tx and Rx antennas along diagonal axes). In these latter cases (Fig. 4.b and 4.d), the sidelobes are reduced and the front-to-back ratio increased.

Fig. 5.b shows the near E-Field intensity distribution and Fig. 5.a indicates the ports used for the S parameters calculations: ports 1 and 3 are out-of-phase RX ports (using an out-of-phase power divider) and ports 2 and 4 are the TX ports. In this case of diagonal positionning of antennas (with coaxial feeding), our simulations confirms that, as for the initial case, the near-field TX contributions are cancelled at the RX points (red points in Fig. 5.b).

The corresponding 3-D radiation pattern also confirms a reduction of the side-lobe level compared to Fig. 2.b.

A theoretical development is proposed in [5] to describe the positive combination in far field and the destructive one in near field using S-parameters between the 4 radiating elements.

As said before, in our case, the first stage of SIC is due to the cross polarization and the second level requires having $\mathrm{S}_{23}$ equals in phase and magnitude to $\mathrm{S}_{21}$ as well as $\mathrm{S}_{41}$

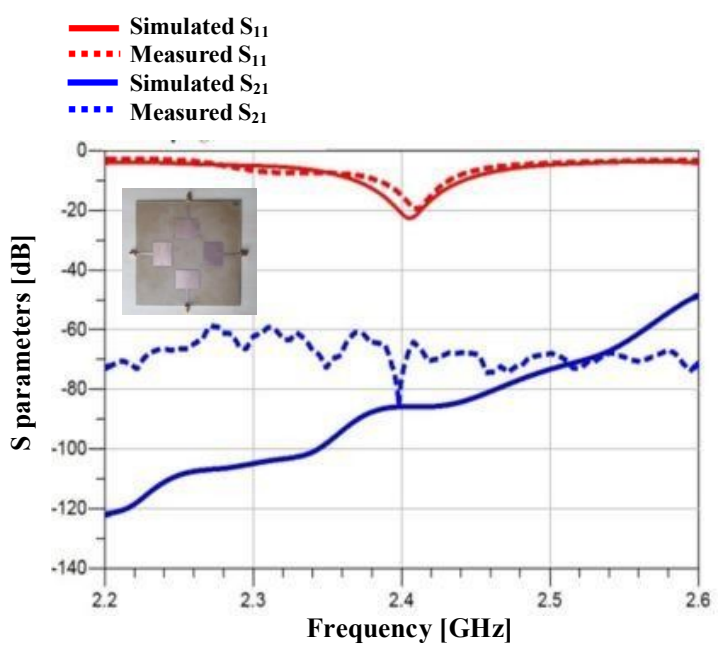

Fig.6 . Simulated and measured input reflection coefficient $\left(\mathrm{S}_{11}\right.$ of Balun+Antennas) and coupling between TX and RX $\left(\mathrm{S}_{21}\right)$ for topology A.

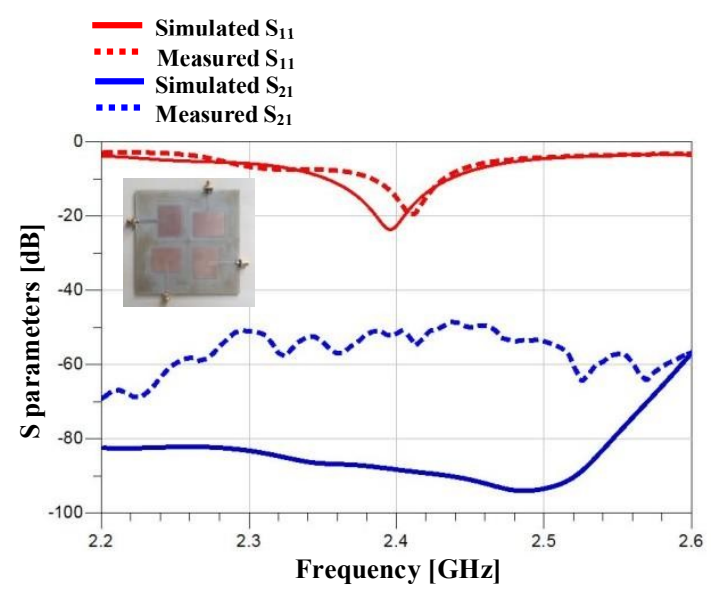

Fig. 7 . Simulated and measured input reflection coefficient $\left(\mathrm{S}_{11}\right.$ of Balun+Antennas) and coupling between TX and RX $\left(S_{21}\right)$ for topology B.

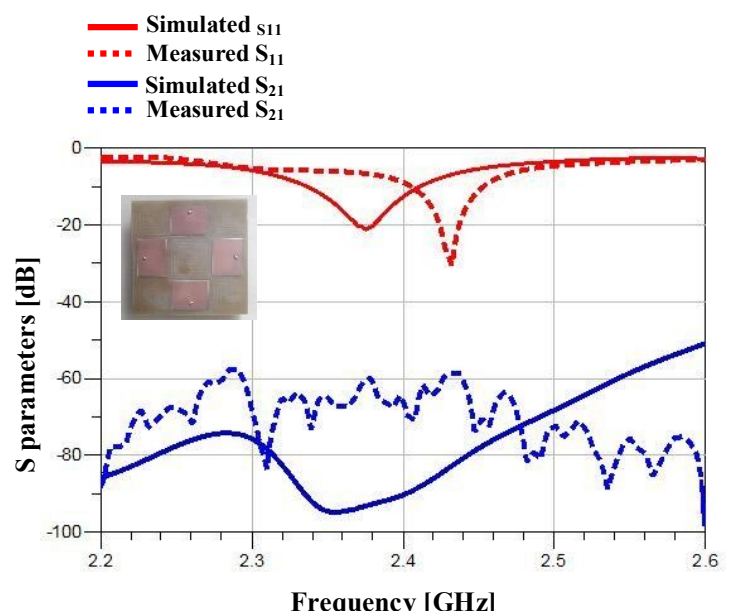

Fig. 8 . Simulated and measured input reflection coefficient $\left(\mathrm{S}_{11}\right.$ of Balun+Antennas) and coupling between TX and RX $\left(\mathrm{S}_{21}\right)$ for topology $\mathrm{C}$. 


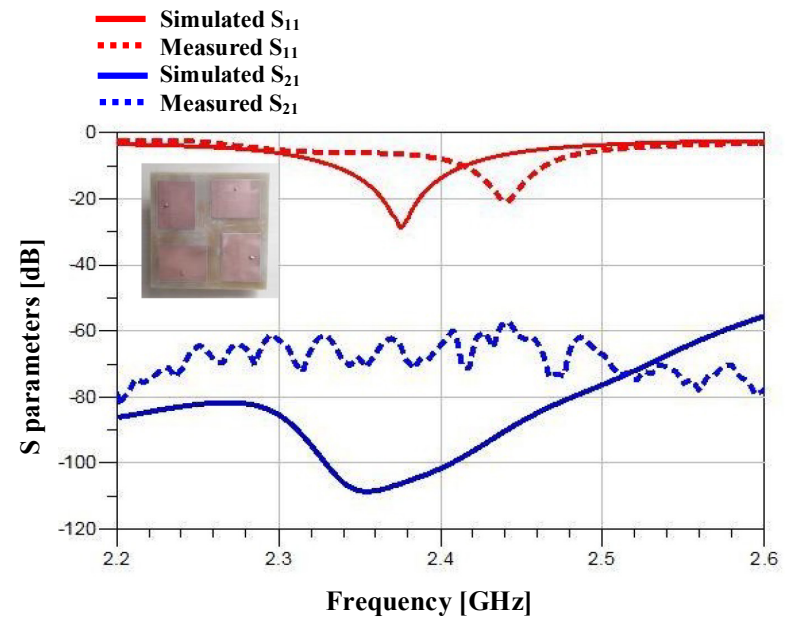

Fig. 9. Simulated and measured Input reflection coefficient $\left(\mathrm{S}_{11}\right.$ of Balun+Antennas) and coupling between TX and RX $\left(\mathrm{S}_{21}\right)$ for topology D.

equals to $\mathrm{S}_{43}$. Nevertheless, that more compact configuration degrades the symmetry between ports 1 and 3 to port 2 for example. The antennas placement has been re-optimized to verify these equalities to insure this near-field cancellation. The final SIC stage uses a balun to merge oppositely the residual components coupled to RX ports 2 and 4 . Here, a diagonal axial symmetry (axis passing through the two RX antenna feeding points) is preserved which guarantees identical S-parameters on each side of this axis (i.e. $\mathrm{S}_{21}=\mathrm{S}_{41}$ and $\left.\mathrm{S}_{23}=\mathrm{S}_{43}\right)$.

Fig. 6 to 9 represent the S-parameters simulated on CST studio and measured using a two-port calibrated Vector Network Analyzer. Reflection coefficient $S_{11}$ as well as $S_{21}$ parameter (which corresponds to the power coupled from TX to RX ports) include two baluns respectively in the TX and RX paths. A first set of measurements has been done by using our self-made baluns and compared to a second run with commercial shielded ones [6]. Coaxial transitions and/or bends and short cables were used between the components to minimize phase and magnitude imbalances and in a such close use the shielded baluns contributed logically to more interesting and reliable SIC values. Then, the measurements are in a rather good agreement with simulations. Nevertheless, slight frequency shifts appear partly because of a small impedance mismatch between the baluns and antennas.

The measured isolation obtained for the first architecture (A) is found to be better than $64 \mathrm{~dB}$ but at the expense of high-level sidelobes. The RX and TX antennas are separated by $6.2 \mathrm{~cm}$ (between the two antenna $50 \Omega$ access-points) with a distance between the TX pair of elements of 7,9 $\mathrm{cm}$ side to side (useful to compute the array factor relatively to the $6.1 \mathrm{~cm}$ half-wavelength at $2.43 \mathrm{GHz}$ ). The top antenna array surface is of about $10,8 \times 10,8 \mathrm{~cm}$. Also, the structure D (Fig. 9) shows an isolation between the two ports better than $58 \mathrm{~dB}$ but for a more compact system. Indeed, the distance between TX and RX feeding points is of $5.4 \mathrm{~cm}$ whereas the $6.3 \mathrm{~cm}$ side to side distance gets close to half-wavelength in the air. The antenna array surface is then of $7.8 \times 7.8 \mathrm{~cm}$.

The experimental set-up was not installed in an anechoic chamber and multipath contributions affected the final isolation results. Then, time-domain measurements were also made using the same 2-ports set-up ( 2 baluns and antennas) in order to identify the indirect paths and the difference of level of the first main direct contribution in each case. The calibrated level of each first contributions follows the same trend as in simulations, which confirms some slight improvements in decoupling thanks to coaxial via feeding and antenna placement. Indeed, the first lobe for configuration $\mathrm{D}$ was measured to be $7 \mathrm{~dB}$ below the one of configuration A. These results are consistent with simulations and confirm indirect path coupling which drives us to plan a new measurement run in an anechoic chamber in order to get closer to the forecast space environment.

\section{CONCLUSION AND FUTURE WORKS}

Four different configurations using low-cost prototyping are studied and SIC is benchmarked for an operating frequency around $2.4 \mathrm{GHz}$. Each system is based on 4 antennas sequentially rotated by $90^{\circ}$. The TX antenna pairs have opposite feeding points and baluns are used to bring equal amplitude and opposite phase to each TX radiating element. A complementary technique is implemented in the RX path. This concept guarantees a high level of SIC at the antenna level together with a classical antenna-array far-field recombination. Moreover, these FD topologies are all able to operate along broadside direction simultaneously for TX and RX. The proposed systematic improvements in the architecture finally lead to topology D with an isolation between two ports better than $58 \mathrm{~dB}$ and for a more compact system that allows reducing sidelobe levels. It has also be shown that coaxial feeding is preferable to microstrip one to avoid spurious coupling. Some preliminary simulations has confirmed that the options considered here together with the compatibility of these topologies with circular polarization seem promising to implement specific FD antenna front-end for Ka-Band ISL link. In that nanosat FD context, focusing our attention on shrinking distance between antennas is of prime interest to develop an antenna network able to reach a high gain both in TX and RX chains.

\section{ACKNOWLEDGMENT}

This work was founded by National Center of Spatial Studies (CNES) and Syrlinks Company. We thank the members for the insightful comments. This project has also been supported by the french Armaments Procurement Agency (DGA), the European Regional Development Fund (ERDF) of the EU, the Brittany Region, the Departmental Council of Finistère and "Brest Métropole" as part of the Cyber-SSI project within the framework of the Brittany 2015-2020 State-Region Contract (CPER)

\section{REFERENCES}

[1] M. Jain,J. Il Choi., T. M. Kim, D. Bharadia, S. Seth,K. Srinivasan, P. Levis, S. Katti, P. Sinha "Practical real-time full duplex wireless", Proc. ACM MobiCom, 2011

[2] E. Aryafar, M. Khojastepour, K. Sundaresan, S. Rangarajan, and M. Chiang "MIDU: Enabling MIMO full

duplex", Proc. ACM MobiCom, 2012.

[3] K. E. Kolodziej, P.T. Hurst, A.J. Fenn, L.I. Parad "Ring array antenna with optimized beamformer for simultaneous transmit and receive",Proc. IEEE APSURSI, 2012

[4] E. Grayver, R. Keating, A. Parower., "Feasibility of full duplex communications for LEO satellite", IEEE Aerospace Conference, 2015.

[5] J. Wu, M. Li, et N. Behdad, « A Wideband, Unidirectional Circularly Polarized Antenna for Full-Duplex Applications ", IEEE Transactions on Antennas and Propagation, vol. 66, n 3, pp. 1559-1563, mars 2018 [6] https://www.markimicrowave.com/Assets/datasheets/BAL0006.pdf? $\mathrm{v}=121$ 